\title{
Application of the Cole-Hopf transformation for finding the analytical solutions of the dynamics of a gravitating system of spherical gas-dust cloud
}

\author{
Mohammed Abobaker \\ Department of Theoretical Mechanics, Institute of Applied Mathematics and Mechanics, St. Petersburg Polytechnic University, Russia.
}

\begin{abstract}
In this paper, we present a simple model for the dynamics of one dimensional of a self-gravitating spherical symmetrical gasdust cloud. We consider two special initial conditions for density and velocity. We take an analytical Cole-Hopf transformation method to study the dynamics of a gravitating system of a gas-dust cloud. The technique is employed to simplify the equations of dynamics, and after that, we applied the method of characteristics to reduce partial differential equations to a system of entirely solvable ordinary differential equations. The obtained results in this study are presented in graphics.
\end{abstract}

Keywords: Hydrodynamics, non-linear PDE, Cole-Hopf method, gravitating system gas-dust cloud.

2010 MSC: 35Q85, 85A05.

(C)2019 All rights reserved.

\section{Introduction}

Dust plays an essential role in the formation of planets. Investigation of planet formation processes has been essential subjects both for astrophysics and for astronomy. To gain an understanding of the planet formation process a model needed to be developed. In this paper, we considered a one-dimensional model of a compressible fluid self-gravitating gas-dust cloud. The gas-dust clouds are extremely complex systems. To enable a theoretical treatment, we have to introduce some simplifications as neglecting rotation, and magnetic fields. By ignoring rotation, and magnetic fields, the cloud can be described as one-dimensional spherically symmetric systems.

The system of equations describing the dynamics of a gravitating medium is mostly non-linear partial differential equations and generally cannot be integrated completely. Therefore, numerical, approximate, and analytical methods are the main way of analyzing such problems. The search for analytical solutions is now motivated by the desire to understand the mathematical structure of the solutions and, hence, a deeper understanding of the physical phenomena described by them.

The dynamics of a gravitating system of gas sphere has received considerable theoretical attention in literature, particularly in connection with the problem of star formation. Larson [7] and Penston [9]

Email address: mhmdbb@yahoo.com (Mohammed Abobaker)

doi: $10.22436 /$ jnsa.012.12.06

Received: 2019-04-20 Revised: 2019-05-23 Accepted: 2019-06-02 
independently found self-similar solutions which describe the density and velocity prior to the instant of protostar formation (defined as the instant at which the central density becomes infinite). Shu [13] extended their work to obtain a whole family of solutions for this problem.

Many numerical simulations have been performed in order to study the dynamics of the collapse of clouds $[1,4,8]$. However numerical methods often do not provide extremely useful information concerning the character of the solution, and an opportunity to understand the internal nature of the solutions obtained. Due to this search for the methods for constructing analytical solutions, will remain one of the important research areas in hydrodynamics.

Considerable attention has been directed towards the study of non-linear problems in hydrodynamics. Apart from a limited number of these problems, most of them do not have an exact solution, so these non-linear equations should be solved using analytical methods [10,11]. For problems of the dynamics of a compressible medium, the hodograph method $[5,10]$ is the most widely used. However, this methods allow obtaining solutions in a specific form of dependence of coordinates and time on fluid flow parameters, which complicates their interpretation and the construction of solutions to initial and boundary-value problems. This method was applied to the study of the formation of the large-scale structure of the universe. As an alternative approach, the Cole-Hopf transformation method [12], in contrast to the Hodograph transformation method allows us to construct solutions either explicitly or in the form of integral of motion, setting the solution in an implicit form.

In this paper, we will study the dynamics of gravitating gas-dust cloud by applying the Cole-Hopf transformation method. The Cole-Hopf transformation provides an attractive method for solving Burger's equation [2]. Studies of the Burgers equation with the help of a transformation discovered by Cole and Hopf [2] can be considered a significant result in hydrodynamics and mathematical physics of the midtwentieth century.

In this work, we employ the method together with the appropriate initial conditions. The technique is used to simplify the equations of dynamics, and then, the method of characteristics is used to reduce the partial differential equation to a system of completely solvable ordinary differential equations. The Cole-Hopf transformation provides an interesting method for solving Burger's equation and can simplify some non-linear partial differential equation and thus makes them analytically solvable.

\section{Fundamental equations}

We consider a spherical gas-dust cloud mass $M$ and radius $R$. Let $p(r, t), \rho(r, t), v(r, t)$, and $\Phi(r, t)$ be the pressure, mass density, radial velocity, and gravitational potential, respectively. We assume spherical symmetry cloud and no rotational motion. In a fluid description, the dynamics of a spherically symmetrical compressible gas-dust cloud is governed by the continuity equation

$$
\frac{\partial \rho}{\partial t}+\rho \frac{\partial v}{\partial r}+\frac{\partial \rho}{\partial r} v+\frac{2}{r} \rho v=0
$$

and the momentum equation

$$
\frac{\partial v}{\partial t}+v \frac{\partial v}{\partial r}+\frac{1}{\rho} \frac{\partial p}{\partial r}=-\frac{\partial \Phi}{\partial r}
$$

The gravitational potential $\Phi$ is given by Poisson's equation

$$
\frac{\partial^{2} \Phi}{\partial r^{2}}+\frac{2}{r} \frac{\partial \Phi}{\partial r}=4 \pi G \rho
$$

where $G$ is Newton's gravitational constant, $p$ the pressure, $\rho$ the density, $v$ the radial velocity, and $\Phi$ gravitational potential. Equations (2.1)-(2.3) are nonlinear partial differential equations are quite complicated, and the general solution cannot be obtained. 


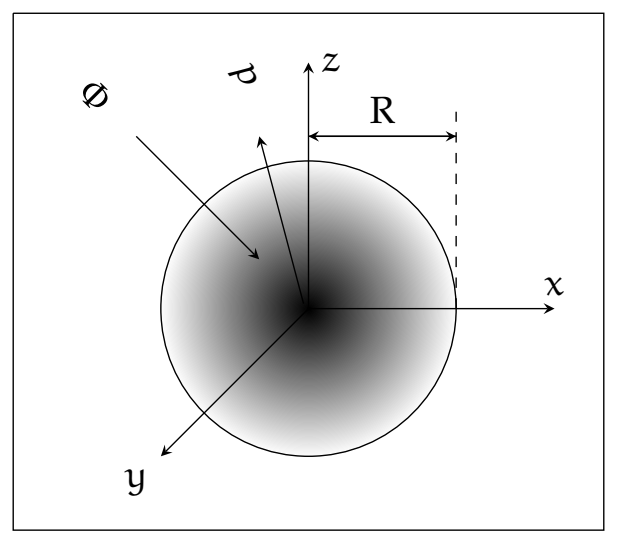

Figure 1: The plot of a spherical symmetric gas-dust cloud, the gravity force $\Phi$ is balanced by pressure force $p$.

We shall simplify our model even further and assume that the cloud collapses as pressure-less dust, which corresponds to the equation of state $p=0$, like many authors have been neglected the pressure to simplify the problem [3, 14]. Let us introduce an auxiliary function $\sigma(r, t)=r^{2} \rho(r, t)$. In this case, we can rewrite the equations (2.1)-(2.3) in this way

$$
\begin{aligned}
\frac{\partial \sigma}{\partial t}+\frac{\partial}{\partial r}(\sigma v) & =0 \\
\frac{\partial v}{\partial t}+v \frac{\partial v}{\partial r} & =-\frac{\partial \Phi}{\partial r} \\
\frac{\partial}{\partial r}\left(r^{2} \frac{\partial \Phi}{\partial r}\right) & =4 \pi G \sigma .
\end{aligned}
$$

\section{Cole-Hopf transformation method}

Many methods for solving nonlinear differential equations were independently suggested during the last decades [6], among these methods Cole-Hopf transformation [2,6]. The Cole-Hopf transformation method provides an interesting approach for solving Burger's equation

$$
\frac{\partial u}{\partial t}+u \frac{\partial u}{\partial r}=v \frac{\partial^{2} u}{\partial r^{2}}
$$

with transformation:

$$
\mathrm{u}(\mathrm{r}, \mathrm{t})=2 \mu \frac{\varphi_{\mathrm{r}}}{\varphi}, \quad \varphi=\varphi(\mathrm{r}, \mathrm{t}) .
$$

Also opened up other doors to solve other non-linear partial differential equations through similar methodologies.

It is a surprising fact that the equations as (2.4)-(2.6) may be solved exactly using a trick discovered independently by Cole and Hopf [2]. After Cole and Hopf introduced the transformation, several attempts have been made to generalized Cole-Hopf transformation; we shall use here modified generalized ColeHopf method $[6,14]$. Let us change the fluid velocity $v$ in the following form:

$$
v(r, t)=-\frac{\theta_{t}}{\theta_{r}}
$$

where $\theta=\theta(r, t)$ is the auxiliary function (generalized Cole-Hopf transformation), $\theta_{t}=\frac{\partial \theta}{\partial t}, \theta_{r}=\frac{\partial \theta}{\partial r}$. The equivalent representation (3.1) has the form of the equation

$$
\theta_{\mathrm{t}}+v(\mathrm{r}, \mathrm{t}) \theta_{\mathrm{r}}=0
$$


Let

$$
v=\mathrm{S}(\mathrm{r}) \mathrm{T}(\theta),
$$

where $S(r)$, and $T(\theta)$ are so far undefined functions. Substitution of (3.3) into the left side of equation (2.5), and using equation (3.2), we obtain

or

$$
\frac{\partial v}{\partial t}+v \frac{\partial v}{\partial r}=S(r) \frac{\partial T}{\partial \theta} \frac{\partial \theta}{\partial t}+S(r) T(\theta)\left(\frac{\partial S}{\partial r} T(\theta)+S(r) \frac{\partial T}{\partial \theta} \frac{\partial \theta}{\partial r}\right),
$$

$$
\frac{\partial v}{\partial t}+v \frac{\partial v}{\partial r}=S^{\prime}(r) S(r) T^{2}(\theta)
$$

We reduce the Poisson equation (2.6) to the form

$$
\frac{\partial \Phi}{\partial r}=4 \pi \mathrm{G} \theta
$$

From equation (2.5), (3.5), and (3.4), we obtain

$$
S^{\prime}(r) S(r) T^{2}(\theta)=-4 \pi G \theta
$$

From (3.6), we obtain

$$
\mathrm{T}^{2}(\theta)=4 \pi \mathrm{G} \theta,
$$

and

$$
S^{\prime}(r) S(r)=-1
$$

Thus, we obtain

$$
\mathrm{T}(\theta)=\sqrt{4 \pi \mathrm{G}} \sqrt{\theta},
$$

and

$$
\mathrm{S}(\mathrm{r})= \pm \sqrt{2} \sqrt{\mathrm{r}^{-1}+\mathrm{c}},
$$

where $c$ is constant of integration. From (3.1), (3.3), (3.8), and (3.9)

or

$$
v(r, t)=-\frac{\theta_{t}}{\theta_{r}}= \pm \sqrt{8 \pi G} \sqrt{r^{-1}+c} \sqrt{\theta},
$$

$$
\theta_{t} \pm \sqrt{8 \pi G} \sqrt{r^{-1}+c} \sqrt{\theta} \theta_{r}=0
$$

By the substitution of new variable

$$
\xi(r)=\int\left(r^{-1}+c\right)^{-1 / 2} d r,
$$

to equation (3.10), it takes the form

$$
\theta_{\mathrm{t}} \pm \sqrt{8 \pi \mathrm{G}} \sqrt{\theta} \theta_{\xi}=0 .
$$

This is a quasi-linear partial differential equation. We can solve it by the method of characteristics [12]. Let us consider the differential form

$$
\frac{\mathrm{dt}}{1}= \pm \frac{\mathrm{d} \xi}{\sqrt{8 \pi \mathrm{G}} \sqrt{\theta}}=\frac{\mathrm{d} \theta}{0},
$$

from (3.13), we have

$$
\theta=c_{1}
$$

where $c_{1}$ is a constant. Also

$$
\xi \pm \sqrt{8 \mathrm{G} \lambda} \sqrt{\theta} \mathrm{t}=\mathrm{c}_{2} .
$$

Combining equations (3.14) and (3.15) we obtain

$$
\mathrm{c}_{2}=\mathrm{F}\left(\mathrm{c}_{1}\right) \text {, }
$$

where $F$ is an arbitrary function, then the general solution to the partial differential equation (3.12) may be written in implicit form

$$
\xi \pm \sqrt{8 \pi G} \sqrt{\theta} t=F(\theta) .
$$




\section{Initial conditions}

One of the main problems with model calculations the formation of planets is the fact that initial conditions of the cloud are not known. We consider in this paper, two initial conditions for the density and velocity. For both conditions, the initial density of fluid as a function of distance vanished at the edge of the cloud and the center has a maximum value. We start with the Cauchy conditions, let us look for the solution to the problem with

$$
\rho(r, 0)=\frac{1}{r^{2}} \frac{\partial \theta_{0}(r)}{\partial r}
$$

where $\theta_{0}(r)=\theta(r, 0)$. Let

$$
\theta_{0}(r)=\rho_{0} \arctan \left(r^{3}\right),
$$

differentiating equation (4.2) and using (4.1), we find $\rho(r, 0)$ (see Figures 2 and 3).

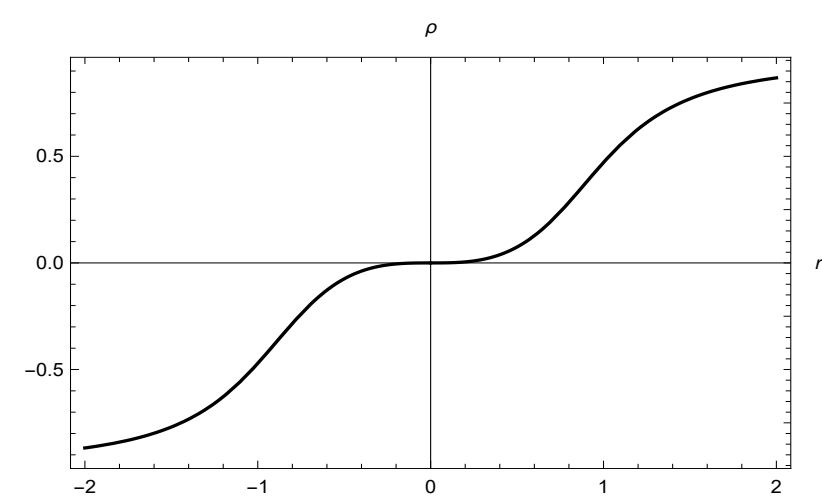

Figure 2: Graph of the inverse tangent function $\theta_{0}$.

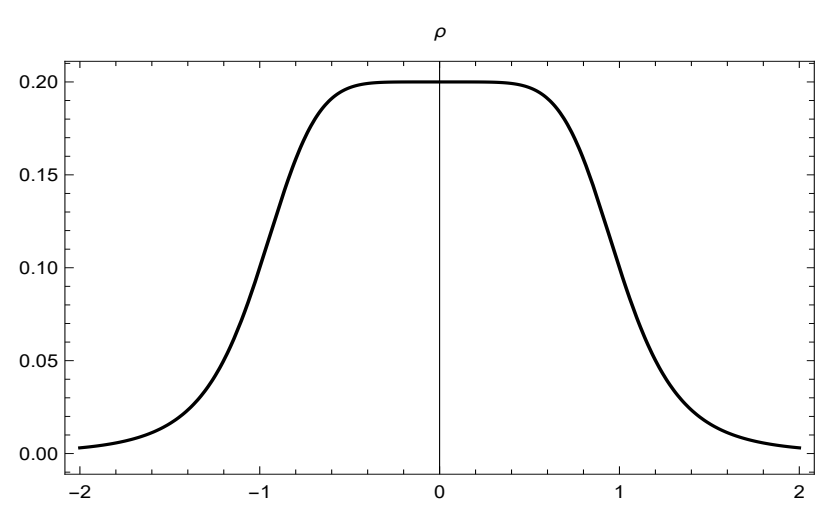

Figure 3: Graph of the initial density function $\rho(r, 0)$.

$$
\rho(r, 0)=\frac{\rho_{0}}{1+r^{6}}>0 .
$$

Assume that velocity of the fluid at the time $t=0$ as following:

$$
v(r, 0)=0 .
$$

The second set of initial conditions are as following:

$$
\theta_{0}(r)=\frac{M}{4 \pi} \operatorname{arsinh}\left(r^{3}\right),
$$

where $\theta_{0}(r)=\theta(r, 0)$, and $M$ is mass of cloud

$$
\rho(r, 0)=\frac{1}{r^{2}} \frac{\partial \theta_{0}(r)}{\partial r}
$$

Differentiating equation (4.5) and using (4.6), we find, $\rho(r, 0)$ (see Figures 4 and 5)

$$
\rho(r, 0)=\frac{3 M}{4 \pi\left(r^{6}+1\right)^{1 / 2}}>0 .
$$

Assume too that velocity of the fluid at the time $t=0$ as

$$
v(r, 0)=0 .
$$




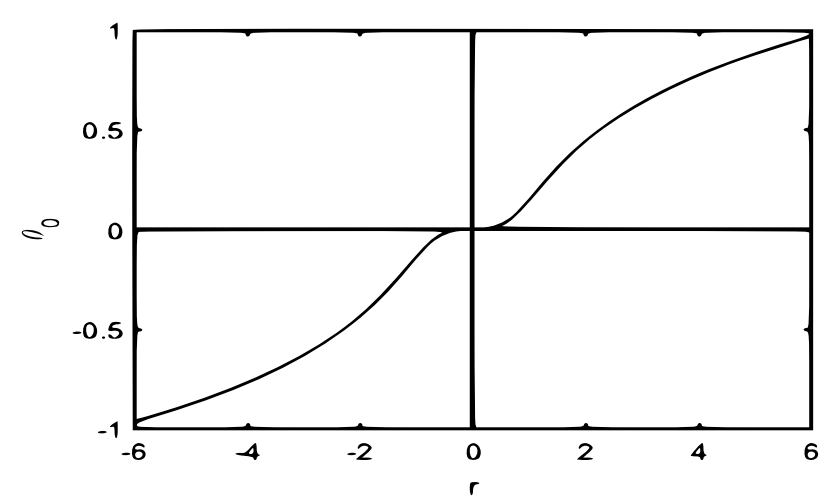

Figure 4: Graph of the inverse hyperbolic sine function, $\theta_{0}(r)=\operatorname{arsinh}\left(r^{3}\right)$.

\section{Analytical solution of fundamental equations}

\subsection{Solution to the problem with the Cauchy conditions}

When $c=0$ in equation (3.11) we obtain

$$
\xi(r)=\frac{2}{3} r^{\frac{3}{2}}
$$

Then we can rewrite equation (4.2) as

$$
\theta_{0}(\xi)=\rho_{0} \arctan \left(\left(\frac{3}{2} \xi\right)^{2}\right) .
$$

We seek now the solution of the equation (3.16) with initial condition (5.2). By plugging initial condition (5.2) into equation (3.16), we get

$$
\mathrm{F}\left(\theta_{0}(\xi)\right)=\xi,
$$

therefore

$$
F(\theta)= \pm \frac{2}{3}\left(\tan \left(\frac{\theta}{\rho_{0}}\right)\right)^{\frac{1}{2}}
$$

Substituting the equation (5.3) into (3.16), we obtain that

$$
\tan \left(\frac{\theta}{\rho_{0}}\right)=\frac{9}{4}(\xi \pm \sqrt{8 \pi \mathrm{G}} \sqrt{\theta} \mathrm{t})^{2},
$$

therefore

$$
\theta=\rho_{0} \arctan \left(\frac{3}{2} \xi \pm 3 \sqrt{2 \pi G} \sqrt{\theta} t\right)^{2},
$$

replacing the variable $\xi$ by its expression from equation (5.1), we finally obtain

$$
\theta-\rho_{0} \arctan (\sqrt{r} \pm \sqrt{2 \pi G} \sqrt{\theta} t)^{2}=0,
$$

which is non-linear inverse trigonometrical transcendental equation, we can solve it numerically to find $\theta$.

Differentiating the equation (5.4) with respect to $r$, we find

$$
\theta_{r}=\frac{3 \rho_{0} \sqrt{\theta} \sqrt{r} w}{\left(\sqrt{\theta}\left(1+w^{4}\right) \pm \rho_{0} \lambda t w\right)}
$$


where

$$
w(r, t)=r^{\frac{3}{2}} \pm \lambda \sqrt{\theta} t, \quad \lambda=3 \sqrt{2 \pi G} .
$$

Now we can calculate density $\rho$ through function $\theta$

$$
\rho(r, t)=\frac{\theta_{r}}{r^{2}}=\frac{3 \rho_{0} \sqrt{\theta}\left(r^{\frac{3}{2}} \pm \lambda \sqrt{\theta} t\right) r^{-\frac{3}{2}}}{\left(\left(1+w^{4}\right) \sqrt{\theta} \pm \rho_{0} \lambda t(\sqrt{r} \pm \lambda \sqrt{\theta} t)\right)} .
$$

Differentiating equation (5.4) with respect to $t$, we obtain

$$
\theta_{\mathrm{t}}=\frac{ \pm 2 \rho_{0} \lambda \theta w(\mathrm{r}, \mathrm{t})}{\sqrt{\theta}\left(1+w^{4}\right) \pm \rho_{0} \lambda \mathrm{tw}} .
$$

Now we can calculate velocity through function $\theta$

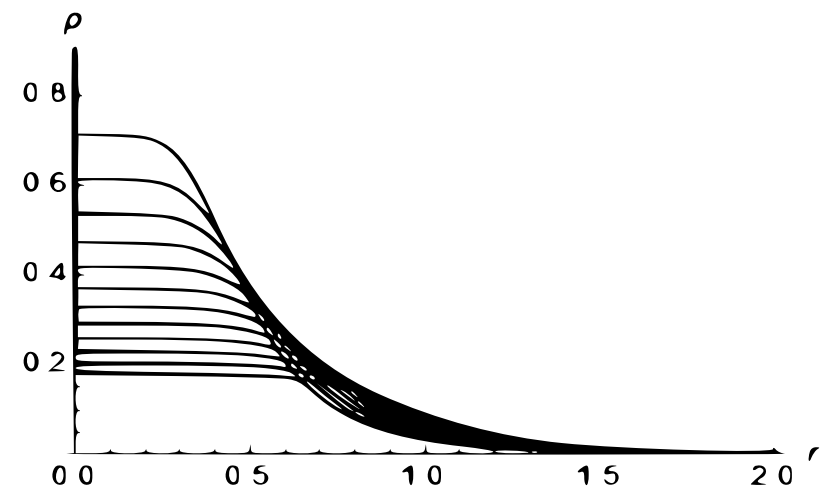

Figure 6: Graphs of density $\rho(r)$ for a variety of time $t$.

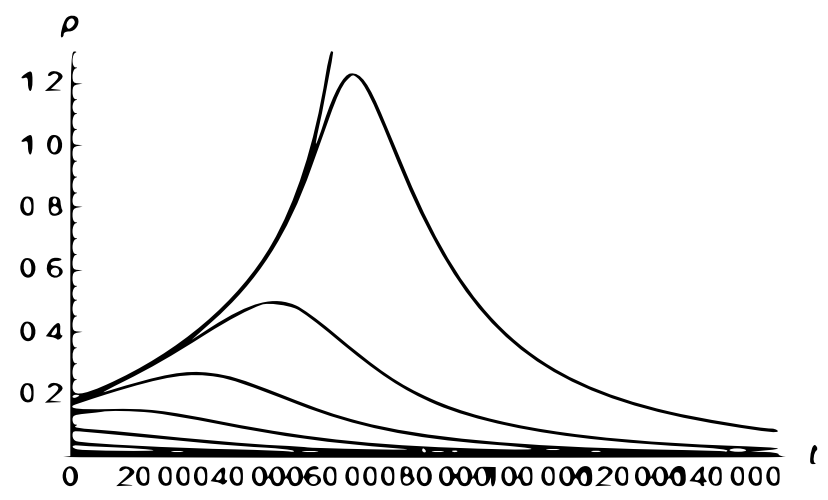

Figure 7: Graphs of density $\rho$ for a variety of radius $r$.

$$
v(r, t)=-\frac{\theta_{t}}{\theta_{r}}= \pm \lambda \theta^{\frac{1}{2}} r^{\frac{1}{2}} .
$$

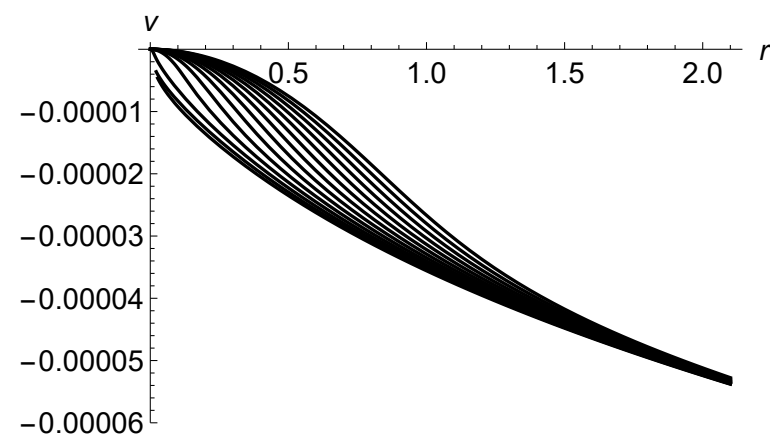

Figure 8: Graphs of velocity $v(r)$ for a variety of times $t$.

\subsection{Solution to the problem with the inverse hyperbolic condition}

Now we want to get the solution of the equation (3.16) with initial condition (4.5), (4.7), and (4.4). The procedure for calculating the function $F(\theta)$ remains similar to that described in the previous subsection 5.1 and reduced to the equation:

$$
\theta-\frac{M}{4 \pi} \operatorname{arsinh}\left(r^{3 / 2} \pm \frac{3}{2} \sqrt{8 \pi G} \sqrt{\theta} t\right)^{2}=0
$$


this is the inverse hyperbolic transcendental equation, and we can solve it numerically to find $\theta$. Differentiating the equation (5.5) with respect to $r$, we find

$$
\theta_{r}=\frac{6 M \sqrt{\theta} w(r, t) \sqrt{r}}{8 \pi \sqrt{\theta} \sqrt{1+w^{4}}-3 M \lambda t w(r, t)},
$$

where

$$
w(r, t)=\left(r^{\frac{3}{2}} \pm \frac{3}{2} \lambda \sqrt{\theta} t\right), \quad \lambda=\sqrt{8 \pi G} .
$$

Now we can calculate density $\rho$ through function $\theta$

$$
\rho=\frac{6 M \sqrt{\theta}\left(r^{\frac{3}{2}} \pm \frac{3}{2} \lambda t \sqrt{\theta}\right) r^{-\frac{3}{2}}}{8 \pi \sqrt{\theta} \sqrt{1+\left(r^{\frac{3}{2}} \pm \frac{3}{2} \lambda \sqrt{\theta} t\right)^{4}}-3 M \lambda t\left(r^{\frac{3}{2}} \pm \frac{3}{2} \lambda \sqrt{\theta} t\right)} .
$$

Differentiating equation (5.5) with respect to $t$, we obtain

$$
\theta_{\mathrm{t}}=\frac{6 \mathrm{M} \theta w(\mathrm{r}, \mathrm{t})}{8 \pi \sqrt{\theta} \sqrt{1+w^{4}}-3 M \lambda t w(r, t)} .
$$

Now we can calculate velocity $v$ through function $\theta$

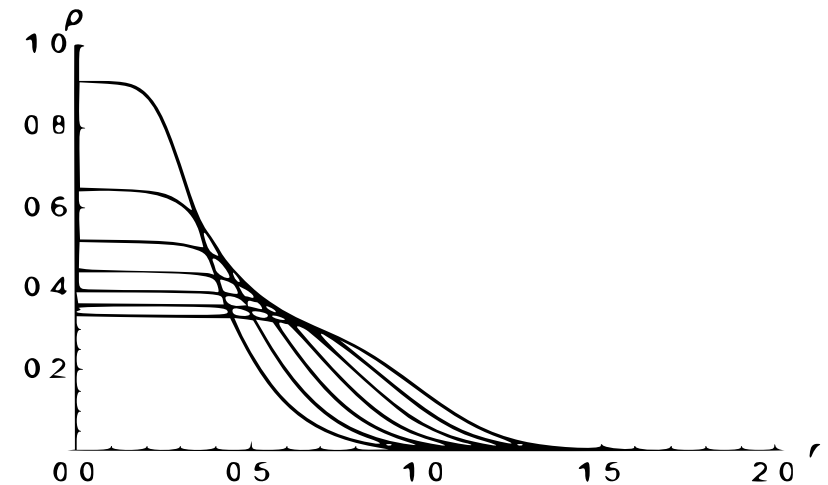

Figure 9: Graphs of density $\rho(r)$ for a variety of times $t$.

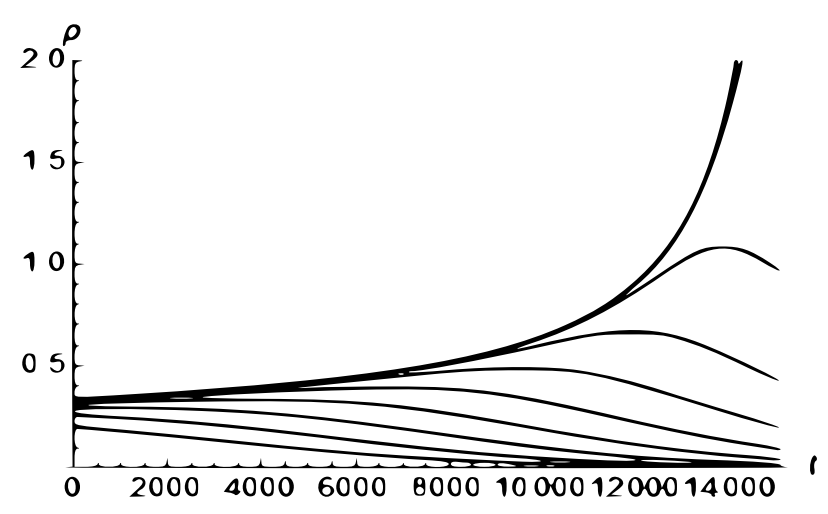

Figure 10: Graphs of density $\rho$ for a variety of radius $r$.

$$
v(r, t)=-\frac{\theta_{t}}{\theta_{r}}=-\frac{\lambda \theta^{\frac{1}{2}}}{r^{\frac{1}{2}}}
$$

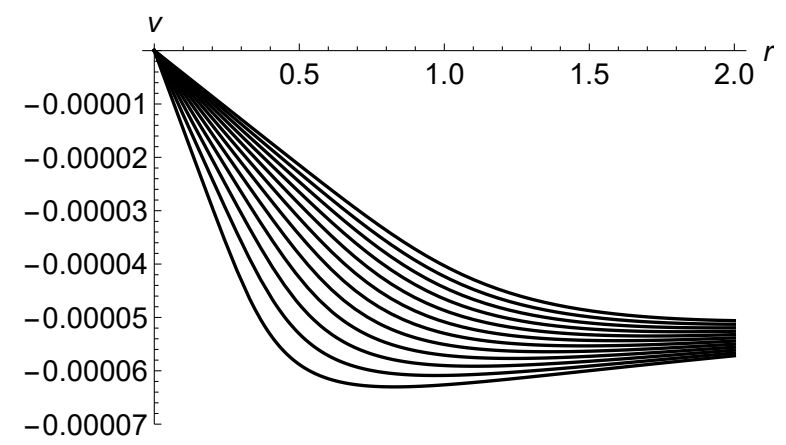

Figure 11: velocity $v(r)$ for a variety of times $t$. 


\section{Results and discussion}

Mathematica package software has been used to solve numerically the inverse trigonometrical transcendental equation (5.4), inverse hyperbolic transcendental equation (5.5), and to generate plots of the density and velocity. The plots start at $r=0.0001$ to avoid infinities at zero, and the real part of the result is used for the plot.

Figures 6 and 8 show the fluid flow variables $\rho$, and $v$ as function of radius $r$ for verity times $t$, as solution for the fundamental equations with special initial condition (4.2), (4.3), and (4.4). It can be observed from graphs, that the density inside the cloud decreases with the increasing value of the distance $r$ from the center of the cloud, whereas the central density inside a cloud increases with increasing time t. Figure 7 shows the density $\rho$ as function of time $t$ for verity of radius $r$.

Moreover, Figure 9 and 11 show the fluid flow variable $\rho$ as function of radius $r$ for verities time $t$ as solution for the fundamental equations with the second special initial conditions (4.5), (4.7), and (4.8). It can be observed from graphs, that the density inside the cloud decreases with the increasing value of the distance $r$ from the centre of the cloud, whereas the central density inside a cloud increases with increasing time $t$. Figure 10 shows the density $\rho$ as function of time $t$ for verity of radius $r$.

\section{Conclusion and future work}

We demonstrated in this paper that the modified form of Cole-Hopf transformation could be used to find the solutions for the dynamics of gravitating spherical symmetrical gas-dust cloud.

In this paper, the Cole-Hopf transformation method has been presented and successfully used to obtain analytic expressions and to illustrate the solutions graphically. We have applied two special initial conditions to get a solution to the problem.

As shown in this paper, the proposed modified Cole-Hopf method, together with the appropriate initial conditions, allow us to get effective scheme to construct a solution to the problem of dynamics gravitating system of the gas-dust cloud. This method allows us to obtain the analytical solution which different to similarity techniques, which have been allowed many authors $[7,9,13]$ to get the semianalytical solution.

A further possible study may be the investigation of the system (2.1)-(2.3) with other possible equation of state, which may be different from the ones considered here and other special initial conditions.

\section{Acknowledgment}

We are thankful to the anonymous referee for useful suggestions and comments for the improvement of the paper.

\section{References}

[1] K. Avinash, B. Eliasson, P. K. Shukla, Dynamics of self-gravitating dust clouds and the formation of planetesimals, Phys. Lett. A, 353 (2006), 105-108. 1

[2] E. Hopf, The partial differential equation $u_{t}+u_{u_{x}}=\mu_{x x}$, Comm. Pure Appl. Math., 3 (1950), 201-230. 1, 3, 3

[3] C. Hunter, The collapse of unstable isothermal spheres, Astrophys. J., 218 (1977), 834-845. 2

[4] E. M. Galimov, A. M. Krivtsov, Origin of the Moon. New Concept: Geochemistry and Dynamics, Walter de Gruyter, Berlin, (2012). 1

[5] A. V. Gurevich, K. P. Zybin, Large-scale structure of the Universe: Analytic theory, Physics-Uspekhi, 38 (1995), 7 pages. 1

[6] N. A. Kudryashov, Analytical theory of non-linear differential equations, Moscow-Izhevsk: Institute of Computer Investigations, Moscow, (2004). 3, 3

[7] R. B. Larson, Numerical calculations of the dynamics of a collapsing proto-star, Monthly Notices of the Royal Astronomical Society, 145 (1969), 271-295. 1, 7

[8] C. Norman, J. Silk, Clumpy molecular clouds- A dynamic model self-consistently regulated by T Tauri star formation, Astrophys. J., 238 (1980), 158-174. 1 
[9] M. V. Penston, Dynamics of self-gravitating gaseous sphere, I. Royal Greenwich Observatory Bulletins, 117 (1966), 299-312. 1, 7

[10] A. D. Polyanin, V. F. Zaitsev, A. I. Zhurov, Methods for solving non-linear equations of mathematical physics and mechanics, Fizmatlit, Moscow, (2005). [in Russian] 1

[11] M. M. Rashidi, A. M. Siddiqui, M. Asadi, Application of homotopy analysis method to the unsteady squeezing flow of a second-grade fluid between circular plates, Math. Probl. Eng., 2010 (2010), 18 pages. 1

[12] P. L. Sachdev, Self-similarity and beyond: exact solutions of nonlinear problems, Chapman and Hall/CRC, New York, (2000). 1, 3

[13] F. H. Shu, Self-similar collapse of isothermal spheres and star formation, Astrophys. J., 214 (1977), 488-497. 1, 7

[14] V. M. Zhuravlev, D. A. Zinov'ev, Non-linear equations linearised using the generalized Cole-Hopf substitutions and the exactly integrable models of the one-dimensional compressible fluid flows, JETP Letters, 87 (2008), 266-270. 2, 3 